\title{
Spatial localization of resistive drift wave structure in tokamak edge plasmas with an embedded magnetic island
}

\section{$\operatorname{AUTHOR}(\mathrm{S}):$}

Hu, Shilin; Li, Jiquan; Qu, Hongpeng; Kishimoto, Y.

\section{CITATION:}

Hu, Shilin ...[et al]. Spatial localization of resistive drift wave structure in tokamak edge plasmas with an embedded magnetic island. Physics of Plasmas 2014, 21(10): 102508.

\section{ISSUE DATE:}

2014-10

URL:

http://hdl.handle.net/2433/191241

\section{RIGHT:}

Copyright 2014 American Institute of Physics. This article may be downloaded for personal use only. Any other use requires prior permission of the author and the American Institute of Physics. 


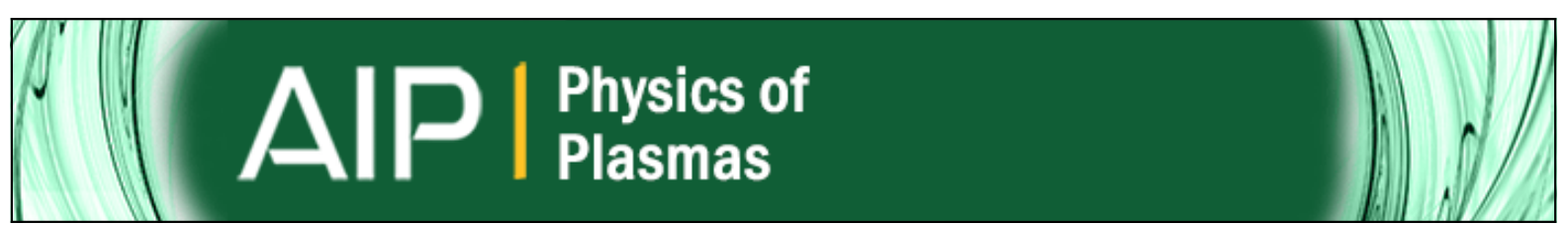

\section{Spatial localization of resistive drift wave structure in tokamak edge plasmas with an embedded magnetic island}

Shilin Hu, Jiquan Li, Hongpeng Qu, and Y. Kishimoto

Citation: Physics of Plasmas (1994-present) 21, 102508 (2014); doi: 10.1063/1.4897942

View online: http://dx.doi.org/10.1063/1.4897942

View Table of Contents: http://scitation.aip.org/content/aip/journal/pop/21/10?ver=pdfcov

Published by the AIP Publishing

\section{Articles you may be interested in}

Development of drift-resistive-inertial ballooning transport model for tokamak edge plasmas

Phys. Plasmas 17, 082511 (2010); 10.1063/1.3478979

A drift-magnetohydrodynamical fluid model of helical magnetic island equilibria in the pedestals of $\mathrm{H}$-mode tokamak plasmas

Phys. Plasmas 17, 062503 (2010); 10.1063/1.3432720

Nonlinear three-mode interaction and drift-wave turbulence in a tokamak edge plasma

Phys. Plasmas 13, 042510 (2006); 10.1063/1.2184291

Wall intersection of ion orbits induced by fast transport of pedestal plasma over an electrostatic potential hill in a tokamak plasma edge

Phys. Plasmas 12, 102501 (2005); 10.1063/1.2052487

Predicting core and edge transport barriers in tokamaks using the GLF23 drift-wave transport model

Phys. Plasmas 12, 052503 (2005); 10.1063/1.1886826

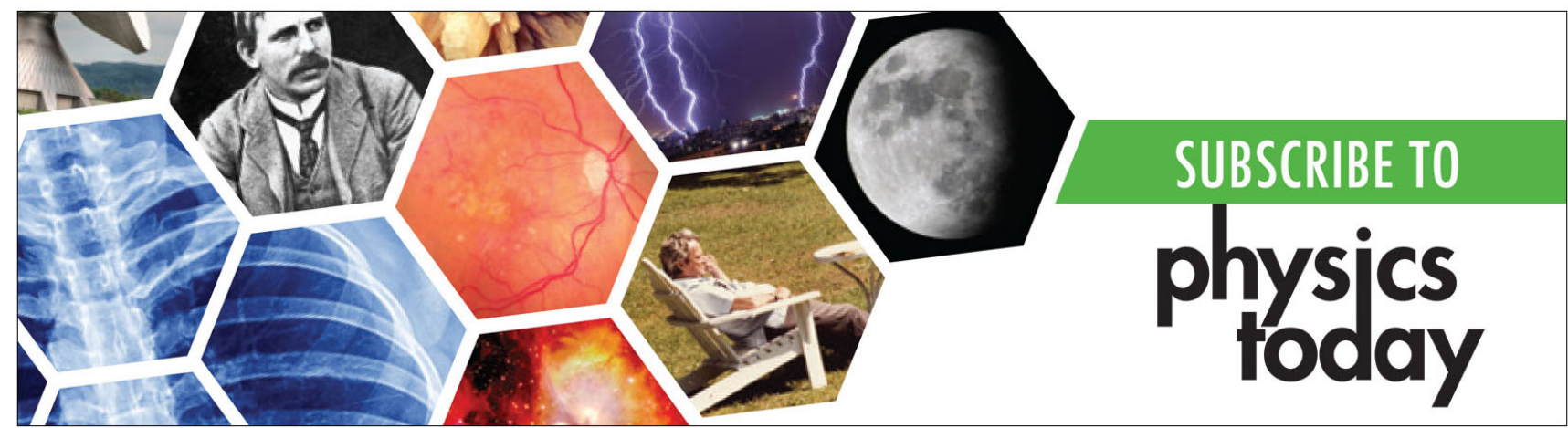




\title{
Spatial localization of resistive drift wave structure in tokamak edge plasmas with an embedded magnetic island
}

\author{
Shilin $\mathrm{Hu},{ }^{1}$ Jiquan $\mathrm{Li}^{2,}{ }^{2, \mathrm{a}}$ Hongpeng Qu, ${ }^{1}$ and $\mathrm{Y}$. Kishimoto ${ }^{2,3}$ \\ ${ }^{1}$ Southwestern Institute of Physics, Sichuan, Chengdu 610041, China \\ ${ }^{2}$ Graduate School of Energy Science, Kyoto University, Gokasho, Uji, Kyoto 6110011, Japan \\ ${ }^{3}$ Institute of Advanced Energy, Kyoto University, Gokasho, Uji, Kyoto 6110011, Japan
}

(Received 24 June 2014; accepted 30 September 2014; published online 16 October 2014)

\begin{abstract}
Resistive drift wave instability is investigated numerically in tokamak edge plasma confined by sheared slab magnetic field geometry with an embedded magnetic island. The focus is on the structural characteristics of eigenmode inside the island, where the density profile tends to be flattened. A transition of the dominant eigenmode occurs around a critical island width $w_{c}$. For thin islands with a width below $w_{c}$, two global long wavelength eigenmodes with approximately the same growth rate but different eigenfrequency are excited, which are stabilized by the magnetic island through two-dimensional mode coupling in both $x$ and $y$ (corresponding to radial and poloidal in tokamak) directions. On the other hand, a short wavelength eigenmode, which is destabilized by thick islands with a width above $w_{c}$, dominates the edge fluctuation, showing a prominent structural localization in the region between the X-point and the O-point of the magnetic island. The main destabilization mechanism is identified as the mode coupling in the $y$ direction, which is similar to the so-called toroidal coupling in tokamak plasmas. These three eigenmodes may coexist in the drift wave fluctuation for the island with a width around $w_{c}$. It is demonstrated that the structural localization results mainly from the quasilinear flattening of density profile inside the magnetic island. (C) 2014 AIP Publishing LLC. [http://dx.doi.org/10.1063/1.4897942]
\end{abstract}

\section{INTRODUCTION}

Magnetic islands are frequently observed in magnetic fusion plasmas. They can be created through MHD instabilities such as various tearing modes including neoclassical tearing mode (NTM), error fields, or externally imposed resonant magnetic perturbations (RMPs). ${ }^{1-7}$ The overlap of large longwavelength islands may cause plasma disruption so that the plasma confinement is deteriorated. Even the dynamics of medium size islands can also give rise to periodic oscillation behavior such as the sawtooth around the rational surface of $q=1$ in tokamak plasmas. On the other hand, it has been realized that the magnetic island may probably benefit the reduction of plasma fluctuations. In this respect, besides the theoretical exploration of the effect of magnetic island on microturbulence, experimental efforts to mitigate or suppress the edge localized modes (ELMs) by external RMPs have been made first in DIII-D, then confirmed in other devices such as JET and ASDEX-U. ${ }^{5-7}$ Even if early theoretical studies on plasma response to the static RMPs have predicted a screening effect of external RMPs produced by plasma rotation, ${ }^{8}$ recent simulations have shown the potentialities of island penetration, at least for some probable parameter windows, ${ }^{9-13}$ besides the experimental efforts. ${ }^{14}$ Hence, the magnetic island dynamics are of importance in plasma equilibrium and transport control as well as the confinement improvement.

In tokamak plasmas, the occurrence of magnetic islands often alters pressure profile, which provides a driving force of short wavelength instabilities, e.g., the drift wave and

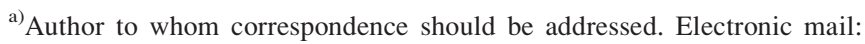
lijq@energy.kyoto-u.ac.jp
}

resistive interchange mode. The island formation and evolution thus occur in a turbulent environment. In fact, different scale fluctuations can be excited by individual driving force and coexist together. They interact nonlinearly and affect each other, probably producing new turbulent structures and transport phenomena. Generally, macroscopic MHD activities with magnetic island dynamics may inevitably lead to global energy confinement degradation. ${ }^{15}$ The magnetic island can cause the plasma profile flattened inside island separatrix due to large parallel transport along the field lines so that the core temperature decreases. The energy confinement thus tends to deteriorate. On the other hand, the global magnetic island structure can give rise to a two-dimensional (2D) mode coupling in both radial and poloidal directions to form a global structure of micro-scale fluctuations. ${ }^{16}$ Such mode coupling transfers fluctuation energy from unstable components to stable or highly damped region, leading to a dissipation of microturbulence. Recently, extensive investigations on the effect of magnetic island on the microinstabilities, e.g., the ion temperature gradient (ITG) mode have been carried out based on fluid (gyrofluid) and gyrokinetic simulations. ${ }^{16-27}$ Theoretical analysis in a gyrokinetic framework is extended for small magnetic islands. ${ }^{28}$ Among complicated linear and nonlinear interaction processes, the mutual relation between the profile relaxation inside island separatrix and the secondary excitation (or destabilization) is an interesting issue. In the multiscale MHD and ITG turbulence, linear analyses of the ITG instability with an initial ion temperature profile with a static magnetic island have showed a separation of rational surface from one into three when the island width becomes larger than a critical threshold, $w_{c}$, leading to a destabilized global ITG mode. Such a 
new ITG mode was referred to as the magnetic island induced ITG mode, namely, the MITG mode. ${ }^{16}$ Though the initial quasilinear flattening of pressure profile was ignored $a$ priori, the predicted MITG has been qualitatively demonstrated in a nonlinear gyrofluid simulation with consistent evolution of dynamic magnetic island where the plasma profile relaxation consistently takes place. ${ }^{27}$

The quasilinear flattening of density profile is also often observed in resistive drift wave turbulence. What role the profile flattening plays in destabilizing the drift wave is a key question. Note that Wilson and Connor have analyzed the effect of equilibrium profile modification by magnetic island based on a rigorous gyrokinetic formalism (hereafter, it is referred to as $\mathrm{W} \& \mathrm{C}$ theory). ${ }^{28}$ A reduction in density and temperature gradients inside the island and a strong $\vec{E} \times \vec{B}$ flow shear around it were observed. Most importantly, it predicted that the drift waves become more localized in the $y$ direction and the position of localized structure is not where the drive for the instability is the strongest. A simplified model, which involves a sinusoidal variation with $y$ in the pressure profile but removes the complication of magnetic island geometry, was proposed to elucidate the underlying mechanism. The same features of the mode structure could be qualitatively reproduced as full model analysis. However, this theory is valid for long, thin islands, with a width comparable to the ion Larmor radius. For thick islands, gyrokinetic analysis is intractable since the magnetic island cannot be treated as a perturbation. Hence, it calls for numerical simulations to examine the analytical formalism and generalize the theoretical framework to large islands.

In this work, we conduct a numerical study on the dissipative drift wave in tokamak edge plasma with an embedded magnetic island to elaborate structural features of the eigenmode as well as instability characteristics. Simulations are performed based on a Hasegawa-Wakatani (HW) model in a slab configuration to limit the effect of profile relaxation on the density only. It is observed that three global eigenmodes may be excited. Thin islands stabilize two long wavelength eigenmodes, but thick islands destabilize a short wavelength one, showing a transition of the dominant eigenmode. The destabilization is identified to mainly result from the mode coupling in the $y$ direction induced by the island. Most importantly, simulations show a spatial localization of the eigenmode structures in the region between the X-point and the O-point of the island, mainly for thick islands. While we have numerically examined the $\mathrm{W} \& \mathrm{C}$ theory, a model with preset quasilinearly flattened density profile inside the magnetic island is proposed to clarify the underlying mechanism of structural localization due to the island effects. Results from modeling analyses are qualitatively in agreement with the simulations as well as the $\mathrm{W} \& \mathrm{C}$ theory.

The remainder of this paper is organized as follows: In Sec. II, the physical model and simulation settings are described. Simulations of the drift wave instability versus the magnetic island width are presented with an emphasis on the features of eigenmode structure in Sec. III. Two modeling analyses are implemented to identify the physical mechanism in Sec. IV. Finally, achieved results are summarized and a brief discussion on the zonal flow dynamics is given in Sec. V.

\section{PHYSICAL MODEL AND SIMULATION SETTING}

Our investigation is implemented in a 2D slab configuration. The equilibrium magnetic field is composed of a guide field $B_{t}$ along the $z$ direction with unit vector $\hat{e}_{z}$ and components in the perpendicular directions ( $x$ and $y$, which correspond to the radial and "poloidal" directions in tokamak, respectively) due to the existence of magnetic island. The resulting magnetic field $\vec{B}$ can be represented as ${ }^{16}$

$$
\vec{B}=B_{t} \hat{e}_{z}+\hat{e}_{z} \times \nabla \psi
$$

where $\psi=\hat{s} x^{2} / 2+\tilde{\psi}(x) \cos \left(k_{T} y\right)$. Here, $\hat{s}$ denotes magnetic shear, $\tilde{\psi}(x)$ the typical profile of the magnetic flux perturbation calculated from the resistive tearing mode, and $k_{T}$ the dominant wave number of the island, which usually corresponds to the component of $m=1$ in the tearing mode. The full island width can thus be estimated through $w \sim 4 \sqrt{\tilde{\psi}(0) / \hat{s}}$ in the "constant- $\tilde{\psi}$ " regime. The assumption of static magnetic island is probably valid since it evolves in a much slower time scale than microturbulence, such as in the Rutherford regime of the tearing mode. Effects of the magnetic island can be incorporated into the drift wave model equations via the parallel operator $\nabla_{/ /}$ with a general expression

$$
\nabla_{/ /} f=\hat{e}_{z} \times \nabla \psi \cdot \nabla_{\perp} f=\bar{\nabla}_{/ /} f+\left[\tilde{\psi} \cos \left(k_{T} y\right), f\right],
$$

where $\bar{\nabla}_{/ /}=\hat{s} x \partial_{y}$ corresponds to the parallel operator in the conventional slab model without magnetic island. The perpendicular differential operator is expressed as $\nabla_{\perp}=\hat{e}_{x} \partial_{x}+$ $\hat{e}_{y} \partial_{y}$ and the Poisson bracket as $[g, f]=\partial_{x} g \partial_{y} f-\partial_{y} g \partial_{x} f$. Here $f$ and $g$ are any equilibrium and/or perturbation quantities, respectively. To highlight the response of density profile to the existence of magnetic island, we use HW turbulence model to describe the evolution of the resistive drift wave in tokamak edge plasma, which consists of the continuity equation for density, $n$, and the equation for vorticity, $\nabla_{\perp}^{2} \phi$ (here $\phi$ is the electrostatic potential). The normalized equations are written as follows: $:^{29,30}$

$$
\begin{gathered}
d_{t}\left[n+\left(L_{n 0} / L_{n}\right) \ln n_{0}\right]=D_{/ /} \nabla_{/ /}^{2}(n-\phi)+\nu_{\perp} \nabla_{\perp}^{2} n, \\
d_{t} \nabla_{\perp}^{2} \phi=D_{/ /} \nabla_{/ /}^{2}(n-\phi)+\mu_{\perp} \nabla_{\perp}^{4} \phi,
\end{gathered}
$$

where $d_{t}=\partial_{t}+\hat{e}_{z} \times \nabla \phi \cdot \nabla$, the normalized coefficient $D_{/ /}$ is inversely proportional to the resistivity $\eta$ as $D_{/ /}=T_{e}\left(\eta n_{0} e^{2} \omega_{c i} L_{n 0} \rho_{s}\right)^{-1}$, namely, inverse normalized resistivity. The parameters $\nu_{\perp}$ and $\mu_{\perp}$ are particle diffusion coefficient and ion viscosity, respectively. Here, $n_{0}$ and $n$ are equilibrium and perturbed electron density, respectively. The time and perpendicular coordinates are normalized as

$$
\omega_{c i} t\left(\rho_{s} / L_{n 0}\right) \rightarrow t ;\left(x / \rho_{s} ; y / \rho_{s}\right) \rightarrow(x ; y) .
$$

Then, the potential and density perturbation are normalized as

$$
\left(L_{n 0} / \rho_{s}\right)\left(e \tilde{\phi} / T_{e}\right) \rightarrow \phi ; \quad\left(L_{n 0} / \rho_{s}\right)\left(\tilde{n} / n_{0}\right) \rightarrow n
$$


Here $L_{n 0}^{-1}=\left|\partial_{x} \ln n_{0}\right|_{x=0}, \rho_{s}=\sqrt{T_{e} / m_{i}} / \omega_{c i}$, and $\omega_{c i}$ is the ion gyroradius. In this work, we focus on the structural features of linear drift wave with an embedded magnetic island. Hence, the linearized HW equations involving the effects of magnetic island are written as

$$
\begin{gathered}
\partial_{t} n+\left(L_{n 0} / L_{n}\right) \partial_{y} \phi=D_{/ /} \bar{\nabla}_{/ /}^{2}(n-\phi)+D_{/ /} \Lambda\left(\tilde{\psi}, k_{T}\right)(n-\phi)+\nu_{\perp} \nabla_{\perp}^{2} n, \\
\partial_{t} \nabla_{\perp}^{2} \phi=D_{/ /} \bar{\nabla}_{/ /}^{2}(n-\phi)+D_{/ /} \Lambda\left(\tilde{\psi}, k_{T}\right)(n-\phi)+\mu_{\perp} \nabla_{\perp}^{4} \phi .
\end{gathered}
$$

The operator $\Lambda\left(\tilde{\psi}, k_{T}\right)$ is expressed explicitly with unfolded Poisson bracket in Eq. (2) as follows:

$$
\begin{aligned}
\Lambda\left(\tilde{\psi}, k_{T}\right)= & \hat{s} x \cos \left(k_{T} y\right)\left(2 \partial_{x} \tilde{\psi} \partial_{y y}^{2}+k_{T}^{2} \tilde{\psi} \partial_{x}\right)+\hat{s} k_{T} \sin \left(k_{T} y\right)\left[\left(\tilde{\psi}-x \partial_{x} \tilde{\psi}\right) \partial_{y}+2 x \tilde{\psi} \partial_{x y}^{2}\right]+k_{T}^{2} \tilde{\psi} \partial_{x} \tilde{\psi} \partial_{x} \\
& +\frac{1}{2}\left[k_{T}^{2} \tilde{\psi}^{2} \partial_{x x}^{2}+\left(\partial_{x} \tilde{\psi}\right)^{2} \partial_{y y}^{2}\right]-\frac{1}{2} \cos \left(2 k_{T} y\right)\left[k_{T}^{2} \tilde{\psi}^{2} \partial_{x x}^{2}-\left(\partial_{x} \tilde{\psi}\right)^{2} \partial_{y y}^{2}\right] \\
& +k_{T} \sin \left(2 k_{T} y\right)\left\{\frac{1}{2}\left[\tilde{\psi} \partial_{x x}^{2} \tilde{\psi}-\left(\partial_{x} \tilde{\psi}\right)^{2}\right] \partial_{y}+\tilde{\psi} \partial_{x} \tilde{\psi} \partial_{x y}^{2}\right\} .
\end{aligned}
$$

Obviously, the magnetic island can not only produce mode coupling in the $x$ direction through the variation of $\tilde{\psi}(x)$ but also couple the components in the $y$ direction through the sine and cosine factors. Furthermore, Eq. (9) also indicates that the magnetic island can induce mode coupling with high order harmonics, which may enhance the effect of large islands on the drift wave instability.

Equations (7) and (8) form a coupling chain of all components in the $y$ direction so that the evolution of the fluctuations can be solved easily as an initial value problem, where Fourier spectral decomposition in the $y$ direction and an implicit finite difference scheme for $x$ variable are employed. ${ }^{19}$ That is, any perturbed quantity $f$ is expressed as

$$
f(x, y, t) \sim \sum_{m} f_{m}(x, t) \exp \left(i 2 \pi m y / L_{y}\right),
$$

with wave number $k_{y}=2 \pi m / L_{y}$. The boundary conditions for all perturbed physical quantities are naturally periodic in the $y$ direction with periodic length $L_{y}$. The fixed boundary condition (usually zero) is employed in the $x$ direction with the simulation box $\left[-L_{x} / 2,+L_{x} / 2\right]$.

\section{SIMULATION FOR EIGENMODE ANALYSIS}

To emphasize the effect of embedded magnetic island, the drift wave instabilities are first simulated in reference parameter regions for direct comparison. Typical numerical parameters in simulations are chosen as: $L_{x}=40$ with grid number of $512, L_{y}=20 \pi$ with mode number of 10-30. The time step is about $\delta t=10^{-3}$. Simulations are started initially with small random perturbation. The initial density profile is set with varied gradient length $L_{n}=L_{n 0} \cosh (2 x / \lambda)$, where finite $\lambda$ can additionally limit radial mode width of the drift wave except for the effect of finite magnetic shear. In this work, usually $\lambda=6$ is taken. The reference physical parameters in simulations are chosen as $\mu_{\perp}=\nu_{\perp}=0.06$, $D_{/ /}=0.05, \hat{s}=0.4$ except for the particular description. Fig. 1 illustrates the parametric dependence of growth rates for different inverse normalized resistivity $D_{/ /}$and magnetic shear $\hat{s}$. The spectral peak of the linear growth rates is located in the region of $k_{y}=0.6-1.1$ and shifts to higher mode number for weak shear or large resistivity. The growth rates decrease as the magnetic shear increases or the normalized resistivity decreases (namely, $D_{/ /} \propto \eta^{-1}$ increases), showing that the magnetic shear plays a stabilizing role and the resistivity destabilizes the resistive drift wave. These results reveal similar parametric dependences of the instability obtained in a shearless slab. ${ }^{30}$ Furthermore, note that an elaborate analytical derivation of the resistive drift wave instability in sheared
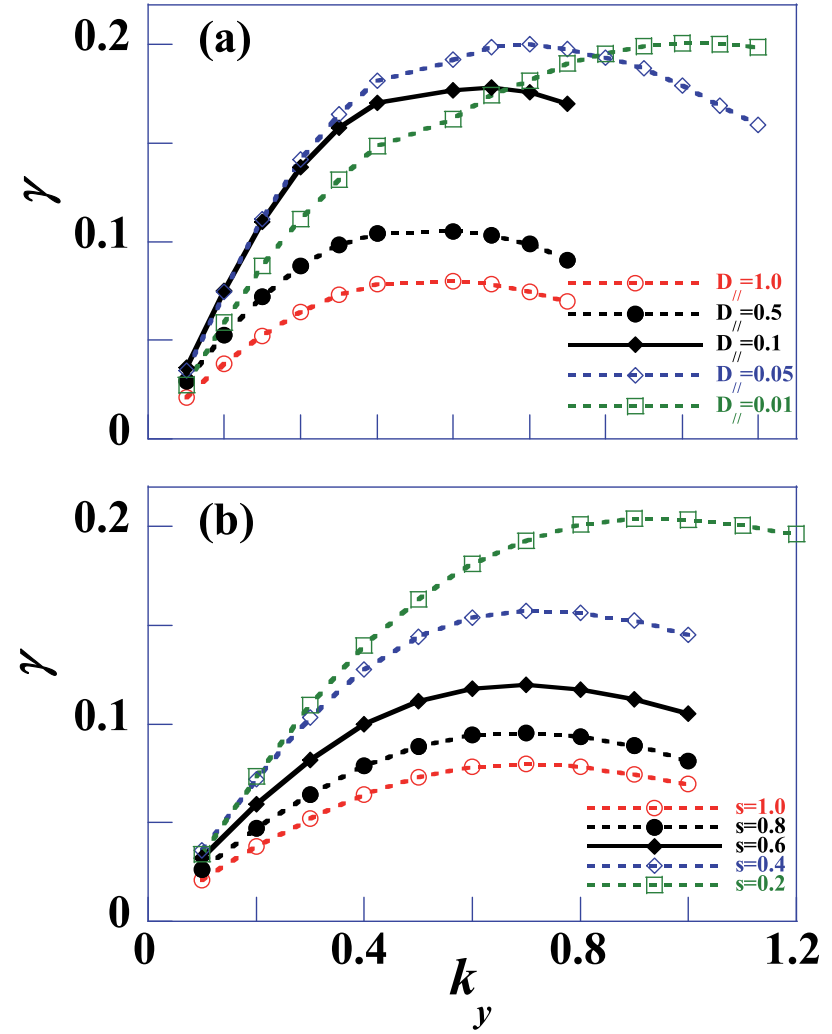

FIG. 1. The growth rate of resistive drift wave versus $k_{y}$ for different dissipation $D_{/ /}$(a) or magnetic shear $\hat{s}$ (b). The reference parameters are used except for $\mu_{\perp}=\nu_{\perp}=0.001, \hat{s}=1.0(\mathrm{a}), D_{/ /}=1.0$ (b). 


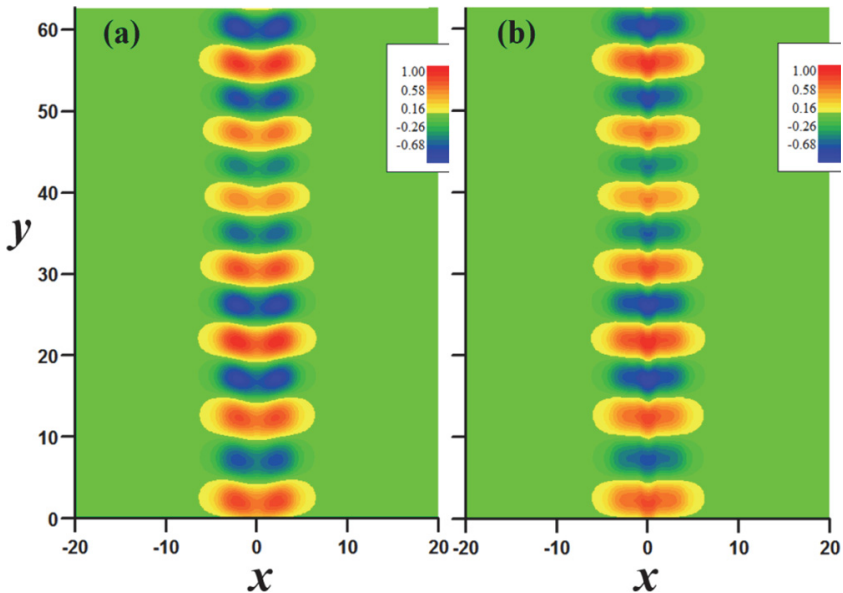

FIG. 2. Contour plots of potential (a) and density (b) perturbations of resistive drift wave in the simulation of Fig. 1. The corresponding parameters are $D_{/ /}=1.0$ and $\hat{s}=1.0$.

slab configuration has been carried out recently, based on a reduced electrostatic two-fluid model. ${ }^{31}$ The dependences of the instability on both the magnetic shear and the resistivity are also qualitatively in agreement with the observations in our simulations. Fig. 2 plots contours of both potential and density perturbations, exhibiting typical mode structures of the resistive drift wave in a sheared slab.

When a magnetic island is embedded in the plasma, it may give rise to some new effects. Typically, the pressure profile (including density and/or temperature) tends to be flattened inside the island separatrix. Furthermore, a new,
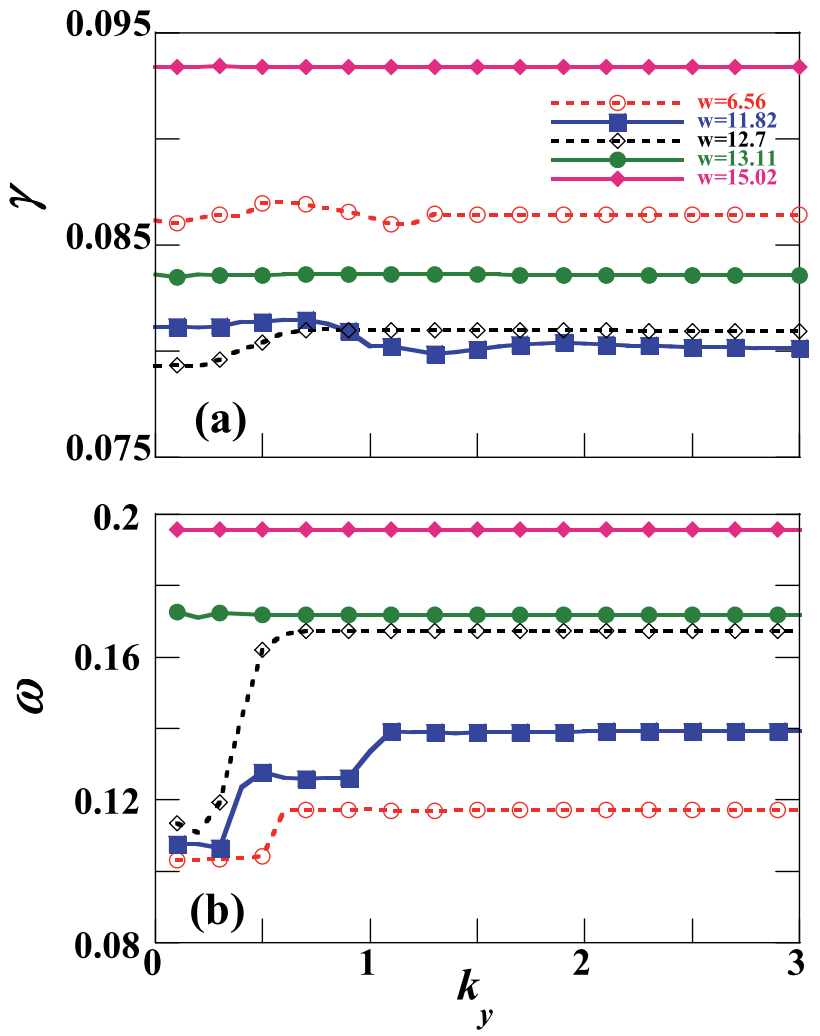

FIG. 3. The growth rate (a) and the eigenfrequency (b) of resistive drift wave versus $k_{y}$ for different island width $w$. The reference parameters are used. destabilized global MITG mode is induced, as described in the Introduction. Moreover, zonal flows can be generated through the linear mode coupling in the $y$ direction due to the existence of magnetic island. Such a mechanism is similar to the toroidal coupling for the excitation of the geodesic acoustic mode (GAM) in a torus like tokamak. ${ }^{32,33}$ To further understand the role of magnetic island in drift wave fluctuations with quasilinear modification of equilibrium profiles, in this work, a static island, which is likely created by a tearing mode, is applied to the equilibrium configuration as a perturbation with the singular layer coinciding with the rational surface.

Simulations are performed with different magnetic island widths. Fig. 3(a) plots the dependence of growth rate on $k_{y}$. For thick islands, all $k_{y}$ components grow with the same growth rate to form a global mode structure, which are similar to the observations in the case of ITG mode. ${ }^{16}$ For thin islands, however, the growth rate versus $k_{y}$ is characterized by more than one stair with small difference. The corresponding eigenfrequency versus $k_{y}$ is illustrated in Fig. 3(b), which is numerically measured during the linear growing phase in the initial value simulation. It can be seen that a single frequency is obtained for all $k_{y}$ components in the cases with thick islands. Meanwhile, two stairs of the eigenfrequency are clearly observed in $k_{y} \leq 0.5$ and $k_{y}>0.5$ regions for thin islands. Furthermore, three stairs appear in $k_{y} \leq 0.3$, $k_{y} \sim 0.5$, and $k_{y} \geq 1.0$ regions for a modest island width. Spectral analyses of fluctuation energy show that in the cases with thin island, the global eigenmodes are characterized by usual resistive drift wave with a spectral peak of fluctuation energy around $k_{y} \sim 0.5$. However, the spectral peak is remarkably shifted to the short wavelength region near $k_{y} \sim$ 1.0 for thick islands, as shown in Fig. 4. These observations seem to suggest a critical island width, $w_{c}$, around which the dominating eigenmode is altered. The stairs of both eigenfrequency and growth rate in Fig. 3 may correspond to different global eigenmode branches. To explicitly illustrate the transition of the dominant eigenmode with the increasing island width, Fig. 5 plots both the growth rate and the

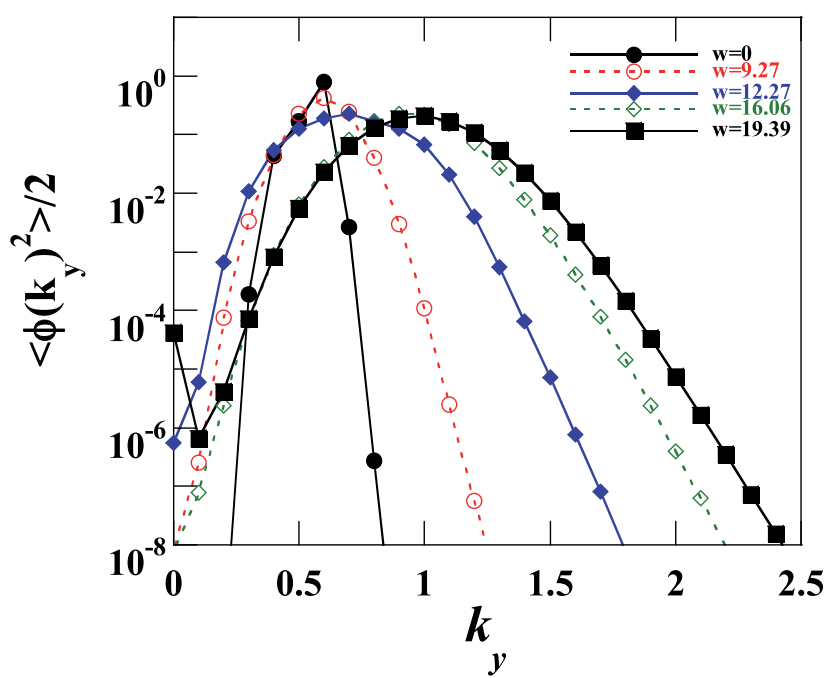

FIG. 4. $k_{y}$ spectra of the mean-square potential $\left\langle\phi\left(k_{y}\right)^{2}\right\rangle / 2$ for different island width $w$. The reference parameters are used. 

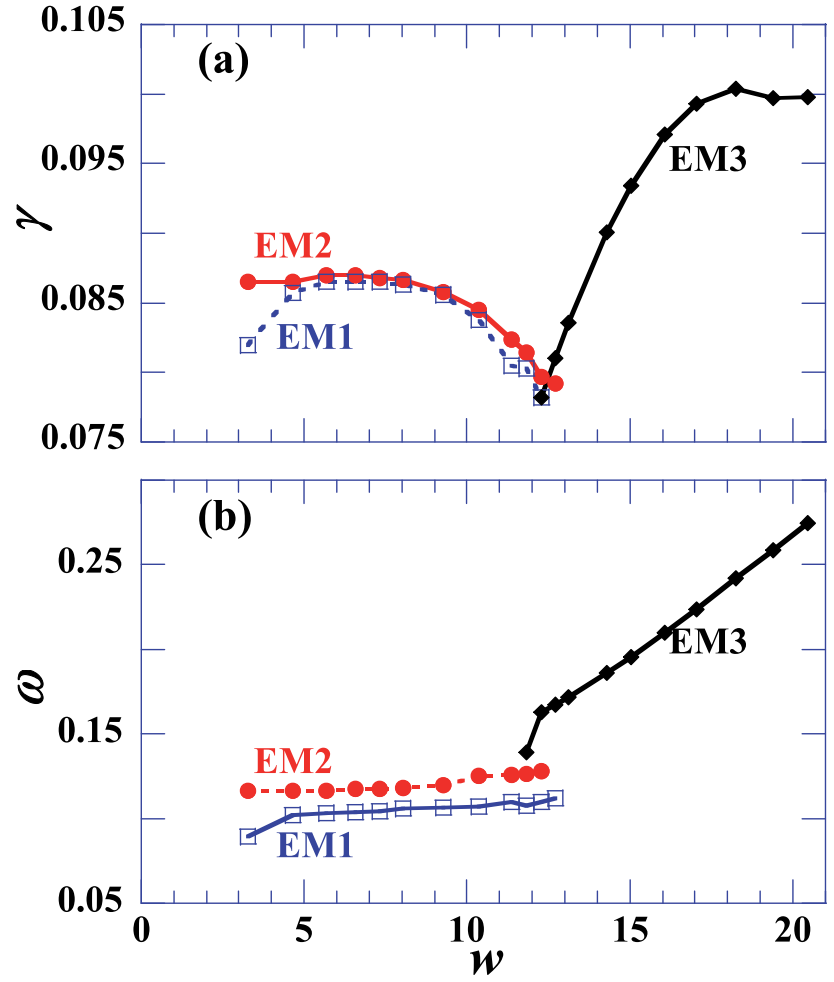

FIG. 5. The growth rate (a) and the eigenfrequency (b) versus magnetic island width $w$. The eigenmodes EM1, EM2, and EM3 correspond to different stairs of the eigenfunction versus $k_{y}$ in Fig. 3(b), respectively. The simulations are the same as in Fig. 3.

eigenfrequency versus the island width. For thin islands, two global eigenmodes with nearly the same growth rate and different eigenfrequency dominate at long wavelengths with $k_{y} \sim 0.5$, labeled as EM1 and EM2, respectively. The thin island has a stabilizing effect on them. To identify the stabilization mechanism, simulations with and without zonal density, which mainly causes the radial flattening of density profile, are performed. Comparison of the growth rates as well as energy spectral analyses shows that the stabilization mainly results from the island-induced 2D mode coupling in the $x$ and $y$ directions since the fluctuation energy of unstable components is noticeably transferred to highly damped region. The zonal density created by linear mode coupling due to the existence of magnetic island has less contribution to the stabilization. However, thick islands destabilize another eigenmode branch (EM3), which dominates the short wavelength fluctuation with $k_{y} \sim 1.0$. This may correspond to the magnetic island induced ITG (namely, MITG) mode observed in linear and nonlinear ITG simulations. ${ }^{16,27}$ The destabilization might originate from two possible mechanisms. One is related to the separation of the rational surface due to thick islands, which is subject to the condition as follows:

$$
k_{/ /} \rightarrow \hat{s} x \partial_{y}+\left[\tilde{\psi} \cos \left(k_{T} y\right), \cdot\right]=0
$$

As analyzed previously in the case of ITG mode, ${ }^{16}$ thick islands can give rise to a separation of rational surface from one $(x=0)$ into three $(x=0$ and the other two neighbors with finite distance) so that a radially global mode with larger growth rate may be excited. ${ }^{34}$ The other mechanism results from the mode coupling in the $y$ direction induced by the magnetic island (hereinafter referred to as $k_{y}$ mode coupling). This kind of mode coupling is quite similar to the socalled toroidal coupling in a torus. It may drive a new, more unstable resistive drift wave, where adjacent $k_{y}$ components couple with each other. However, in this case all $k_{y}$ components are excited along the same rational surface rather than the individual rational surface for each $k_{y}$ component as in the case of toroidal coupling. Hence, it could be inferred that the dependence of the destabilizing effect of thick islands on the radial parametric variations is relatively weak. This inference will be further discussed in Sec. IV.

Note that the $k_{y}$ mode coupling should destabilize the resistive drift wave regardless of the island width, similar to the toroidal coupling in a torus. To properly clarify the effect of the $k_{y}$ mode coupling in the simulations with thin islands, we suggest that the $k_{y}$ mode coupling may play dual roles in influencing the resistive drift wave. One is to stabilize the drift wave through transferring the fluctuation energy from unstable long wavelength components to highly damped region. The stabilization effect acts mainly on the long wavelengths and tends to be stronger as the width of thin islands increases. The other is the destabilizing effect due to a new driving mechanism, which acts mainly on the short wavelengths. The competition between strong stabilizing and weak destabilizing effects of thin islands could lead to an overall stabilization of long wavelength eigenmodes EM1 and EM2. If the destabilizing effect dramatically increases with increasing the width of thick islands, strong destabilization could dominate the effect of thick islands on the short wavelength eigenmode EM3, as shown in Fig. 5.

The stabilization or destabilization of resistive drift wave by magnetic island also depends on the plasma parameters such as dissipation and magnetic shear. Fig. 6 plots the maximum growth rate versus the island width for different dissipation (corresponding to the resistivity) or magnetic shear. Note that the growth rates of different eigenmodes (EM1 and EM2) are slightly different as shown in Fig. 3(a) although they appear in different $k_{y}$ regions. Here, the $k_{y}$ dependence of growth rate, thus, should be weak, particularly for thick islands. That means, the growth rate for a given island width in Fig. 6 roughly corresponds to all $k_{y}$ components. As the resistivity $\eta$ increases (namely, $D_{/ /} \propto \eta^{-1}$ decreases) or the magnetic shear decreases, the stabilizing effect of thin islands tends to be weak, but the destabilizing effect of thick islands is enhanced. Furthermore, the resistivity does not seem to influence the critical island width $w_{c}$, as shown in Fig. 6(a). Meanwhile, Fig. 6(b) indicates that the magnetic shear can enlarge $w_{c}$ effectively. This seems to be consistent with the argument that the critical magnetic island width $w_{c}$ may be mainly governed by the separation of the rational surface. ${ }^{16}$

Most interestingly, the existence of magnetic island noticeably alters the structural features of resistive drift wave. The eigenmode structure is globally shaped by thin islands but it is localized evidently in the region between the $\mathrm{X}$-point and the O-point for thick islands, as shown in Fig. 7. The visualization of eigenmode structure based on mode 

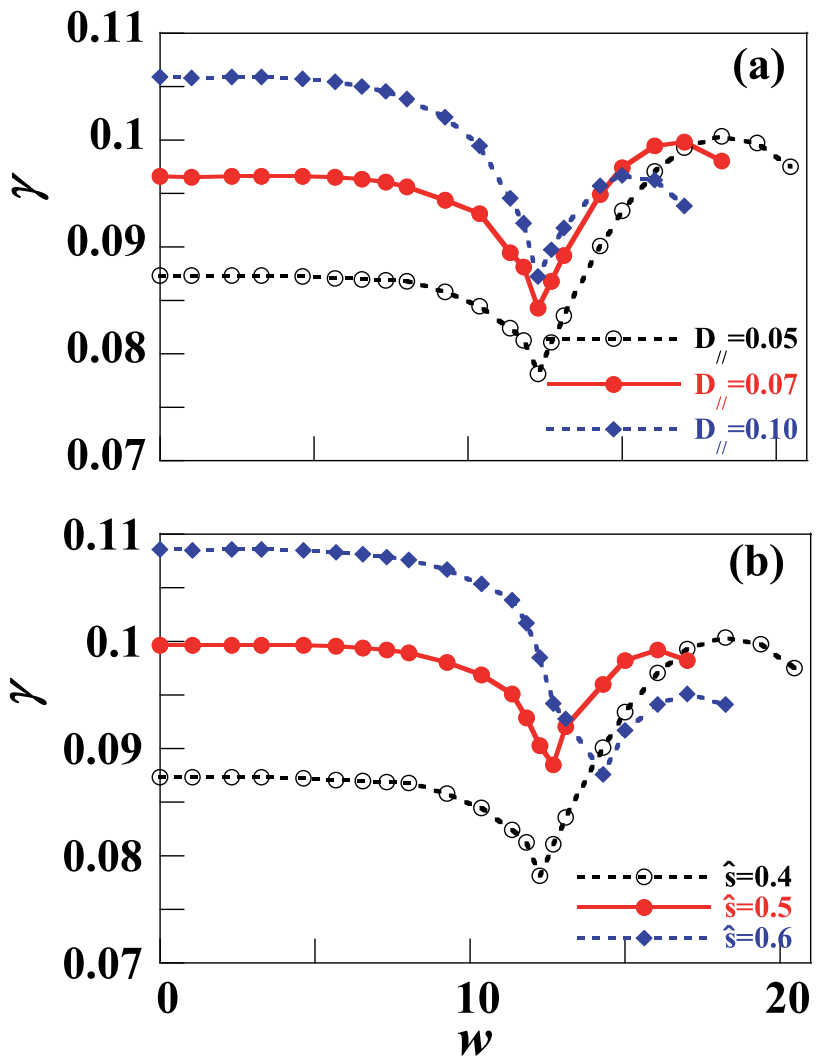

FIG. 6. The growth rate of resistive drift wave versus the magnetic island width $w$ for different dissipation $D_{/ /}$(a) or magnetic shear $\hat{s}$ (b). The reference parameters are used except for $\hat{s}=0.4$ (a), $D_{/ /}=0.05$ (b).

decomposition shows that the island-shaped global structure induced by a thin island in Fig. 7(a) is dominated by the components $k_{y}=0-0.5$ of eigenmode branches EM1 and EM2. Other short wavelength components exhibit a similar structure to usual resistive drift wave, as shown in Fig. 2. For thick islands, the localized eigenmode structure in Fig. 7(c) is mainly constituted of the short wavelength components of $k_{y} \geq 1.0$. Most prominently, the EM2 branch with $k_{y}=$ 0.4-0.9 dominates the island-shaped global structure for the island with a width below $w_{c}$. The EM1 branch at long wavelengths of $k_{y}=0.0-0.3$ and the EM3 branch at short wavelengths of $k_{y} \geq 1.0$ are characterized by the usual structure of resistive drift wave, as plotted in Fig. 8, showing less

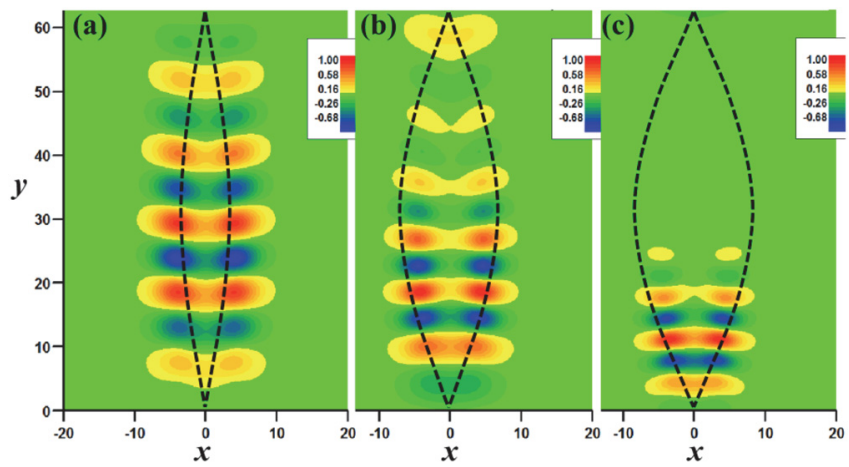

FIG. 7. Contour plots of the potential of resistive drift wave for different island width $w$. Here, $w=5.68$ (a), $w=11.82$ (b), $w=13.11$ (c). The dashed curves plot the separatrix of corresponding magnetic islands. The simulations are the same as in Fig. 3.

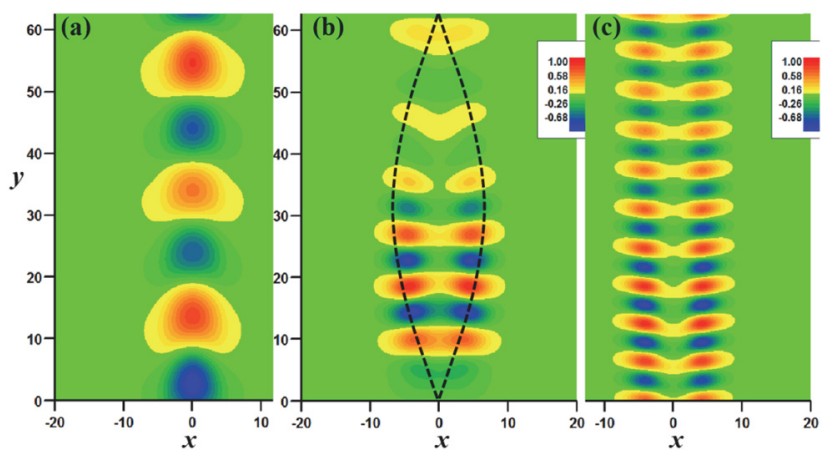

FIG. 8. Contour plots of the decomposed potential with different $k_{y}$ components of $k_{y}=0-0.3$ (a), $k_{y}=0.4-0.9$ (b), $k_{y}=1.0-3.0$ (c) in the simulation with $w=11.82$. The dashed curve plots the separatrix of the corresponding magnetic island. The simulations are the same as that in Fig. 3.

influence of magnetic island. To elucidate the origin of the structural localization due to thick islands, we propose modeling analyses with a focus on the effect of density flattening due to the existence of magnetic island.

\section{MODELING ANALYSES}

Note that in the W\&C theory under the approximation of thin magnetic island, an analytical prediction on the structural localization of ITG eigenmode in the region between the $\mathrm{X}$-point and the O-point has been confirmed by WKB analysis by ascribing the major role of magnetic island in the ITG mode to the profile flattening of density or temperature. ${ }^{28}$ However, the structural localization in Fig. 7(c) is enhanced by thick islands. To ascertain the underlying mechanism of the structural localization observed in resistive drift wave, we first examine the analytical model presented in the $\mathrm{W} \& \mathrm{C}$ theory, and then we propose a numerical model to evaluate the role of profile flattening of density inside a magnetic island in determining the eigenmode structure.

\section{A. Analytical model}

In the $\mathrm{W} \& \mathrm{C}$ theory, the effect of profile flattening was mainly replaced by an equilibrium gradient model with a sinusoidal variation with $y$ but without proper magnetic island geometry, i.e., $k_{/ /} \rightarrow \hat{s} x \partial_{y}$. Similarly, we assume that the density profile is modified by the island as follows:

$$
L_{n}(x, y)=L_{n 0}\left[1+\varepsilon \cos \left(K_{y} y\right)\right] \cosh (2 x / a) .
$$

As a result, the diamagnetic drift frequency becomes a bivariate function of $(x, y) .{ }^{21}$ Here, $\varepsilon$ is a numerical amplitude factor to measure the island effect, which corresponds to the island width. The wave number of magnetic island structure, $K_{y}$, corresponds to the most unstable component $m=1$ of the tearing mode. By incorporating this new profile in Eqs. (7) and (8), simulations are carried out using the same parameter setting as in Fig. 3 except for the amplitude factor $\varepsilon$. Fig. 9 plots $k_{y}$ dependences of both eigenfrequency and growth rate for different $\varepsilon$. A distribution of both eigenfrequency and growth rate versus $k_{y}$ is observed with more than one stair for small $\varepsilon$, similar to the counterpart in Fig. 3 for thin islands. The $k_{y}$ spectra of fluctuation energy versus $\varepsilon$ 

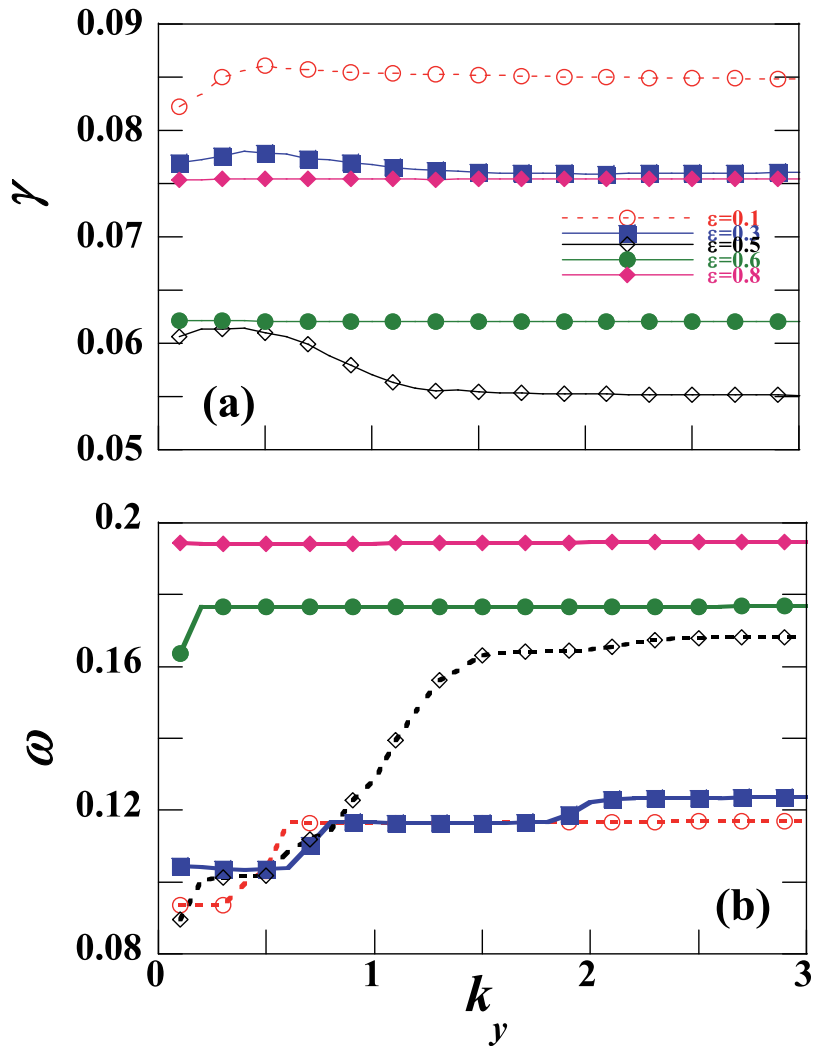

FIG. 9. The growth rate (a) and the eigenfrequency (b) versus $k_{y}$ for different factor $\varepsilon$. The magnetic island has been removed in the configuration in these simulations. The reference parameters are used.

are plotted in Fig. 10. The spectral peak shifts from the long wavelength of $k_{y} \approx 0.5$ to the shorter wavelength of $k_{y} \approx 1.0$, with increasing $\varepsilon$. A critical amplitude factor occurs at $\varepsilon_{c} \approx 0.5$, around which the eigenmodes EM1, EM2, and EM3 coexist and the transition of dominating eigenmode may take place, as shown in Fig. 11. Obviously, the variation of $L_{n}$ in the $y$ direction for small factor $\varepsilon$ stabilizes two long wavelength eigenmodes, EM1 and EM2. Meanwhile, large factor $\varepsilon$ can destabilize the short

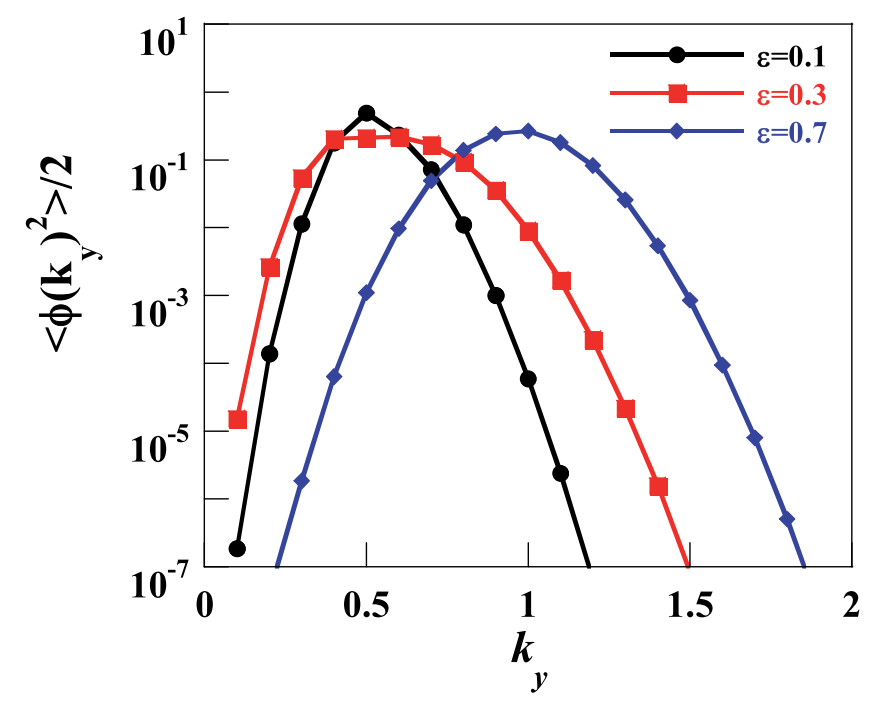

FIG. 10. $k_{y}$ spectra of the mean-square potential $\left\langle\varphi\left(k_{y}\right)^{2}\right\rangle / 2$ for different amplitude factor $\varepsilon$. The simulations are the same as those in Fig. 9 .
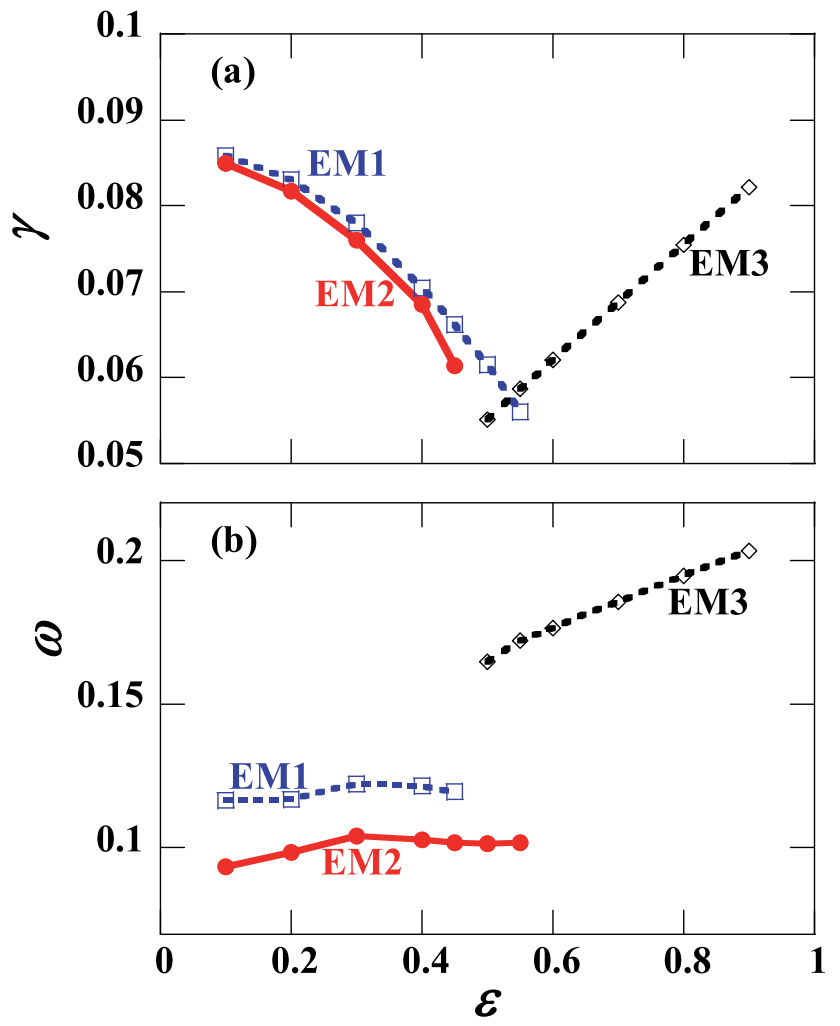

FIG. 11. The growth rate (a) and the eigenfrequency (b) versus the amplitude factor $\varepsilon$. The eigenmodes EM1, EM2, and EM3 correspond to different stairs of the eigenfunction versus $k_{y}$ in Fig. 9(b), respectively. The reference parameters are used.

wavelength eigenmode EM3. The features of structural localization due to strong variation of $L_{n}$ in the $y$ direction, i.e., for large $\varepsilon$, are well revealed as shown in Fig. 12, which is similar to Fig. 7(c). Direct comparison indicates that the effect of magnetic island on the linear resistive drift wave is probably represented mainly by the effect of density variation in the $y$ direction.

\section{B. Numerical model}

Generally, profile flattening inside the magnetic island is a common phenomenon in plasmas. Although the modeling,

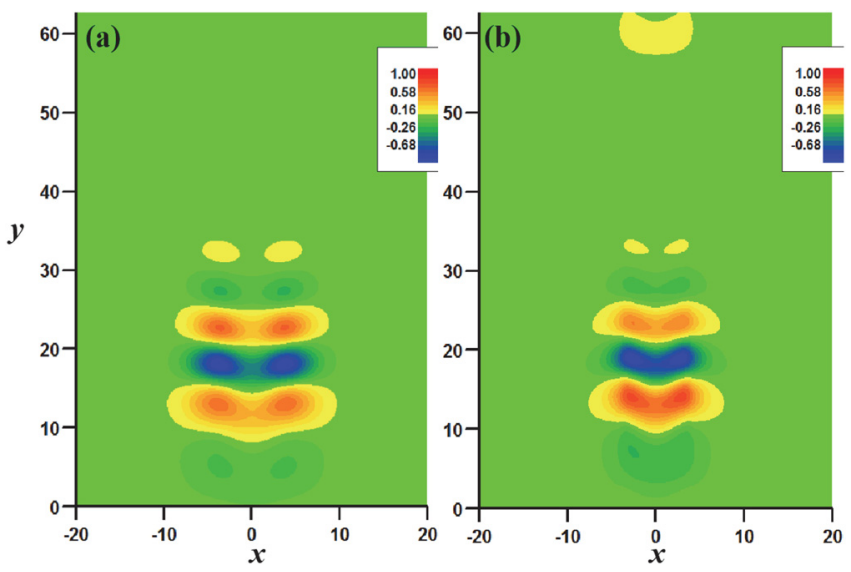

FIG. 12. Contour plots of potential (a) and density (b) perturbations of the resistive drift wave in the simulation with $\varepsilon=0.5$. The simulation is the same as that in Fig. 9. 
Eq. (12), has captured the variation of density profile in the $y$ direction due to the existence of magnetic island, however, it has missed to express profile flattening in the radial direction inside the island, which usually reduces the driving force of the drift wave instability. To examine the role of this mechanism, we incorporate the island effect in resistive drift wave through two separate steps. First, an island-shaped density profile is established by solving density diffusion problem in the configuration with a magnetic island, i.e., $k_{/ /} \rightarrow \hat{s} x \partial_{y}+\left[\tilde{\psi} \cos \left(k_{T} y\right), \cdot\right]$. The drift wave is, then, simulated based on a new equilibrium with the island-shaped density profile but without the magnetic island, i.e., $k_{/ /} \rightarrow \hat{s} x \partial_{y}$. The effect of magnetic island is artificially replaced by an island-shaped density profile in this step. This numerical model may treat more realistic plasma response to the magnetic island, where at least the radial flattening of the density has been incorporated properly.

The establishment of island-shaped density profile is accomplished through solving Eq. (2) without any electrostatic potential fluctuations. To match the parametric setting in the simulations above, an initial density profile with local radial gradient around the rational surface $x=0$ only is approximately reconstructed as $n_{0}=-0.5 x+10.0$. Here, the factor $\cosh (2 x / \lambda)$ in the density gradient length has been ignored since it is important mainly in the region near the rational surface. The profile relaxation of density inside the magnetic island takes place as a transport problem in the simulation. Finally, a steady-state equilibrium density with island-shaped profile is established as shown in Fig. 13. Obviously, the profile in the $x$ direction is flattened in the Opoint region corresponding to $y=10 \pi$, whilst the profile near the X-point corresponding to $y=0$ almost keeps the initial gradient. Note that a model family of flattened profiles for different local diamagnetic frequency was used to quantify the stabilizing effect of magnetic island with carrying out a 2D stability analysis, showing a similar feature of island-shaped profile. $^{21}$ Simulations have demonstrated that

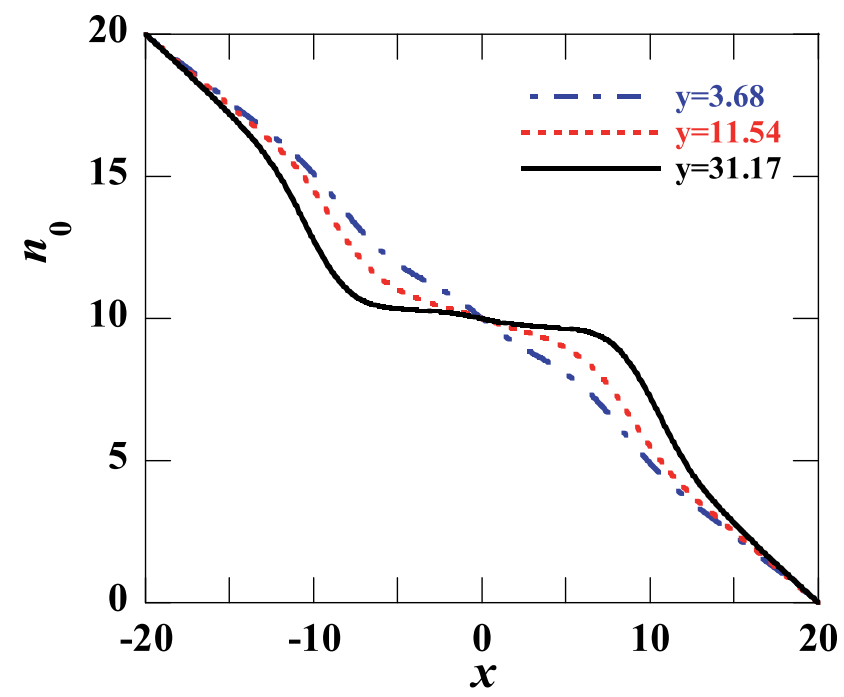

FIG. 13. Radial profiles of density in the quasi-steady state equilibrium for different $y$. Here, $y=0$ and $y=10 \pi$ correspond to the X-point and the Opoint of magnetic island, respectively. The reference parameters are used except for $D_{/ /}=0.5, w=13.11$.

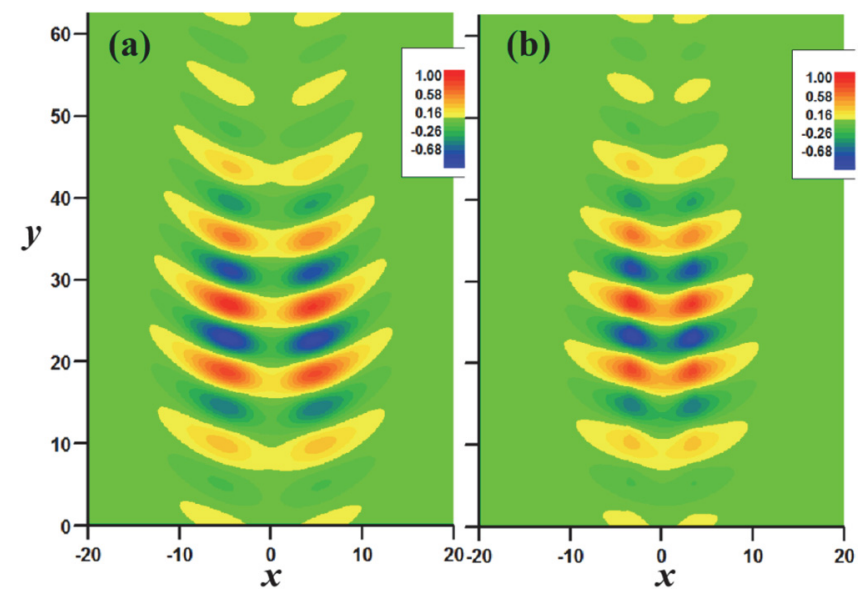

FIG. 14. Contour plots of potential (a) and density (b) perturbations in the simulation with an initial island-shaped density profile. The reference parameters and $w=18.25$ are used to establish the initial equilibrium density.

the profile flattening tends to be more prominent as the parameter $D_{/ /}$increases, namely, for large parallel diffusivity.

Based on such a new, island-shaped equilibrium density profile, the resistive drift wave instability is simulated again in the configuration without magnetic island, i.e., $k_{/ /} \rightarrow \hat{s} x \partial_{y}$. Results show that the island-shaped density profile can also produce strong $k_{y}$ mode coupling. The $k_{y}$ spectral peak of fluctuation energy is located around $k_{y} \approx 0.7$. Most importantly, the structural localization of drift wave eigenmode and its position are reproduced, as shown in Fig. 14, which are similar to those in the simulations with an embedded magnetic island. Note that the localized eigenmode in this numerical model is globally extended comparing with Fig. 7(c) for thick islands. It, instead, looks to be similar to that as shown in Fig. 7(a) for thin islands. This discrepancy may be partially ascribed to the 2-step treatment of the island effect. The island-shaped density profile established in the first step may be canceled again by the density fluctuations in the second step since it has lost support of the magnetic island. Furthermore, comparison among the results from realistic simulations, analytical model, and numerical model above indicates that the position of localized structure seems to result mainly from the variation of density profile in the $y$ direction between inside and outside magnetic island. The density flattening in the radial direction inside the island may not play a major role except for the reduction of the growth rate of drift wave instability.

Finally, it is worthwhile further briefly discussing the destabilization mechanism of the magnetic island. Comparing the destabilizing effect of thick islands in both realistic simulations with magnetic island as shown in Fig. 5 and the modeling analyses with density variation in the $y$ direction, which results from the existence of magnetic island, as shown in Fig. 11, we may conclude that the main destabilization mechanism can be ascribed to the $k_{y}$ mode coupling based on the similarity of the results. Although the separation of rational surface due to thick islands can provide a destabilizing mechanism, which drives a radially global mode instability, it is still weaker than the destabilizing effect due to the $k_{y}$ mode coupling since the separation of 
rational surface induced by thick magnetic islands does not occur in the modeling analyses.

\section{SUMMARY}

In this work, the resistive drift wave instability in tokamak edge plasma with an embedded static magnetic island is simulated focusing on the spatial characteristics of the eigenmode structure. Simulations are performed based on Hasegawa-Wakatani equations in slab geometry to elaborate the underlying mechanism. The magnetic island makes mode coupling of the resistive drift waves in both $x$ and $y$ directions. As a result, the $k_{y}$ spectral distributions of both eigenfrequency and growth rate are characterized by more than one stair in which each stair corresponds to an individual global eigenmode. It is shown that a transition of dominating eigenmode occurs around a critical island width $w_{c}$. Two long wavelength global eigenmodes with approximately the same growth rate but different frequency are stabilized by thin islands mainly through $2 \mathrm{D}$ mode coupling. On the other hand, a dominant short wavelength eigenmode is destabilized by thick islands with a width above $w_{c}$, showing a structural localization in the region between the X-point and the O-point of the magnetic island. These three eigenmodes may coexist for the island width around $w_{c}$. To clarify the underlying physical mechanisms, an analytical model of profile variation in the $y$ direction based on the $\mathrm{W} \& \mathrm{C}$ theory is examined and a numerical model incorporating radial flattening of density inside the magnetic island is proposed. It is demonstrated that the destabilizing mechanism of thick magnetic islands is mainly ascribed to the $k_{y}$ mode coupling induced by the magnetic island besides a weak destabilizing effect resulting from the separation of rational surface. The structural localization of eigenmodes due to thick islands is identified to originate from the quasilinear flattening of density profile due to the existence of magnetic island.

Finally, it is worth-mentioning that the zonal flows can be created through the mode coupling produced by magnetic islands even in the linear drift wave. The generation mechanism is similar to the GAM in a torus, which is excited through the toroidal coupling. Simulations have shown that the zonal flow in linear drift wave is quite weak when the island width is small, i.e., below the critical island width $w_{c}$. However, it is observed that the zonal flow is dramatically enhanced by a large magnetic island, as shown in Fig. 4 for $w=19.39$. Although such large island width may invalidate the approximation of assuming the magnetic island as a perturbation in the equilibrium, still it is an interesting observation since the zonal flow dynamics is of importance in the edge turbulence as in the core plasma with magnetic island. ${ }^{35}$ The zonal flows may be driven by the synergetic effect of both linear and nonlinear mode coupling. Such an issue on the zonal flow dynamics in nonlinear resistive drift wave with a magnetic island is left for a future work.

\section{ACKNOWLEDGMENTS}

This study was supported by National Natural Science Foundation of China Grant Nos. 11175057 and 11205053 , National Magnetic Confinement Fusion Science Program under Grant Nos. 2011GB105002, 2013GB111005, and 2013GB107000. This study was also partially supported by a Grant-in-Aid from JSPS (No. 26400531).

${ }^{1}$ H. P. Furth, J. Killeen, and M. N. Rosenbluth, Phys. Fluids 6, 459 (1963).

${ }^{2}$ A. I. Smolyakov, Plasma Phys. Controlled Fusion 35, 657 (1993).

${ }^{3}$ R. Fitzpatrick, Phys. Plasmas 5, 3325 (1998).

${ }^{4}$ F. L. Waelbroeck, Nucl. Fusion 49, 104025 (2009).

${ }^{5}$ T. E. Evans, R. A. Moyer, K. H. Burrell, M. E. Fenstermacher, I. Joseph, A. W. Leonard, T. H. Osborne, G. D. Porter, M. J. Schaffer, P. B. Snyder, P. R. Thomas, J. G. Watkin, and W. P. West, Nat. Phys. 2, 419 (2006).

${ }^{6}$ Y. Liang, H. R. Koslowski, P. R. Thomas, E. Nardon, B. Alper, P. Andrew, Y. Andrew, G. Arnoux, Y. Baranov, M. Becoulet, M. Beurskens, T. Biewer, M. Bigi, K. Crombe, E. De La Luna, P. de Vries, W. Fundamenski, S. Gerasimov, C. Giroud, M. P. Gryaznevich, N. Hawkes, S. Hotchin, D. Howell, S. Jachmich, V. Kiptily, L. Moreira, V. Parail, S. D. Pinches, E. Rachlew, and O. Zimmermann, Phys. Rev. Letts. 98, 265004 (2007)

${ }^{7}$ W. Suttrop, T. Eich, J. C. Fuchs, S. Günter, A. Janzer, A. Herrmann, A. Kallenbach, P. T. Lang, T. Lunt, M. Maraschek, R. M. McDermott, A. Mlynek, T. Pütterich, M. Rott, T. Vierle, E. Wolfrum, Q. Yu, I. Zammuto, H. Zohm, and ASDEX Upgrade Team, Phys. Rev. Lett. 106, 225004 (2011).

${ }^{8}$ R. Fitzpatrick and T. C. Hender, Phys. Fluids B 3, 644 (1991).

${ }^{9}$ M. Becoulet, F. Orain, P. Maget, N. Mellet, X. Garbet, E. Nardon, G. T. A. Huysmans, T. Casper, A. Loarte, P. Cahyna, A. Smolyakov, F. L. Waelbroeck, M. Schaffer, T. Evans, Y. Liang, O. Schmitz, M. Beurskens, V. Rozhansky, and E. Kaveeva, Nucl. Fusion 52, 054003 (2012).

${ }^{10}$ F. L. Waelbroeck, I. Joseph, E. Nardon, M. Becoulet, and R. Fitzpatrick, Nucl. Fusion 52, 074004 (2012).

${ }^{11}$ F. Orain, M. Becoulet, G. Dif-Pradalier, G. Huijsmans, S. Pamela, E. Nardon, C. Passeron, G. Latu, V. Grandgirard, A. Fil, A. Ratnani, I. Chapman, A. Kirk, A. Thornton, M. Hoelzl, and P. Cahyna, Phys. Plasma 20, 102510 (2013).

${ }^{12}$ A. Monnier, G. Fuhr, P. Beyer, F. A. Marcus, S. Benkadda, and X. Garbet, Nucl. Fusion 54, 064018 (2014).

${ }^{13}$ M. F. Heyn, I. B. Ivanov, S. V. Kasilov, W. Kernbichler, P. Leitner, V. V. Nemov, W. Suttrop, and the ASDEX Upgrade Team, Nucl. Fusion $\mathbf{5 4}$ 064005 (2014)

${ }^{14}$ T. E. Evans, D. M. Orlov, A. Wingen, W. Wu, A. Loarte, T. A. Casper, O. Schmitz, G. Saibene, M. J. Schaffer, and E. Daly, Nucl. Fusion 53, 093029 (2013).

${ }^{15}$ Z. Chang and J. D. Callen, Nucl. Fusion 30, 219 (1990).

${ }^{16}$ Z. X. Wang, J. Li, Y. Kishimoto, and J. Q. Dong, Phys. Plasmas 16, 060703 (2009)

${ }^{17}$ C. J. McDevitt and P. H. Diamond, Phys. Plasmas 13, 032302 (2006).

${ }^{18}$ A. Ishizawa and N. Nakajima, Phys. Plasmas 14, 040702 (2007).

${ }^{19}$ F. Militello, F. L. Waelbroeck, R. Fitzpatrick, and W. Horton, Phys. Plasmas 15, 050701 (2008).

${ }^{20}$ J. Li, Y. Kishimoto, Y. Kouduki, Z. X. Wang, and M. Janvier, Nucl. Fusion 49, 095007 (2009).

${ }^{21}$ F. L. Waelbroeck, F. Militello, R. Fitzpatrick, and W. Horton, Plasma Phys. Controlled Fusion 51, 015015 (2009).

${ }^{22}$ E. Poli, A. Bottino, and A. G. Peeters, Nucl. Fusion 49, 075010 (2009).

${ }^{23}$ M. Muraglia, O. Agullo, S. Benkadda, M. Yagi, X. Garbet, and A. Sen, Phys. Rev. Lett. 107, 095003 (2011).

${ }^{24}$ W. A. Hornsby, A. G. Peeters, M. Siccinio, and E. Poli, Phys. Plasmas 19, 032308 (2012)

${ }^{25} \mathrm{~J}$. Li and Y. Kishimoto, Phys. Plasmas 19, 030705 (2012).

${ }^{26}$ P. P. Hilscher, K. Imadera, J. Li, and Y. Kishimoto, Plasma Fusion Res. 8, 2403040 (2013)

${ }^{27}$ J. Li, Y. Kishimoto, and Z. X. Wang, Phys. Plasmas 21, 020703 (2014).

${ }^{28} \mathrm{H}$. R. Wilson and J. W. Connor, Plasma Phys. Controlled Fusion 51, 115007 (2009)

${ }^{29}$ B. D. Scott, H. Biglari, P. W. Terry, and P. H. Diamond, Phys. Fluids B 3 , 51 (1991).

${ }^{30}$ S. J. Camargo, D. Biskamp, and B. D. Scott, Phys. Plasmas 2, 48 (1995).

${ }^{31}$ F. Militello, M. Ottaviani, and A. Wynn, Phys. Plasmas 21, 022115 (2014).

${ }^{32}$ N. Winsor, J. L. Johnson, and J. M. Dawson, Phys. Fluids 11, 2448 (1968).

${ }^{33}$ K. Miki, Y. Kishimoto, N. Miyato, and J. Li, Phys. Rev. Lett. 99, 145003 (2007).

${ }^{34}$ J. Li, W. X. Qu, L. Huang, and J. H. Zhang, Phys. Lett. A 233, 85 (1997).

${ }^{35}$ M. Leconte and P. H. Diamond, Phys. Plasmas 19, 055903 (2012). 